PROCEEDINGS OF THE

AMERICAN MATHEMATICAL SOCIETY

Volume 133, Number 11, Pages 3403-3409

S 0002-9939(05)07888-3

Article electronically published on May 2, 2005

\title{
ENTROPY-CONSTRAINED FUNCTIONAL QUANTIZATION OF GAUSSIAN PROCESSES
}

\author{
SIEGFRIED GRAF AND HARALD LUSCHGY
}

(Communicated by Richard C. Bradley)

\begin{abstract}
The sharp asymptotics for the entropy-constrained $L^{2}$-quantization errors of Gaussian measures on a Hilbert space and in particular, for Gaussian processes is derived. The condition imposed is regular variation of the eigenvalues of the covariance operator.
\end{abstract}

\section{INTRODUCTION AND RESULTS}

The quantization of probability distributions is an old story which starts in the late 1940's. It has been conceived in order to drastically cut down the storage of signal data to be analysed. For a comprehensive survey of the theory of quantization including its historical development we refer to Gray and Neuhoff [6]. For the mathematical aspects of quantization, one may consult Graf and Luschgy [5] and for more applied aspects in the field of information theory and signal processing, the book of Gersho and Gray [4] is appropriate.

Rigorous extensions of the high-rate result, where the rate is measured by the number of quantization levels (the so-called fixed-rate problem) to the functional quantization of continuous time stochastic processes, have been established for the Gaussian case. See Luschgy and Pagès [10, [11. Recently, the high-rate behaviour of quantization when the rate is measured by the entropy of the quantizers has attracted new attention in finite dimensions. (See Gray et al. [7]). We extend the finite-dimensional setting to entropy-constrained functional quantization of continuous time stochastic processes. The main result of this paper is the sharp asymptotics of the entropy-constrained $L^{2}$-quantization error for a large class of Gaussian processes in a Hilbert space setting.

The framework can be stated as follows. Let $(B,\langle\cdot, \cdot\rangle)$ be a real separable Hilbert space with norm $\|\cdot\|=\langle\cdot, \cdot\rangle^{1 / 2}$ and let $X$ be a centered Gaussian $B$-valued random vector. Let $\mathcal{F}$ denote the set of quantizers $f$ of $X$, that is, $f: B \rightarrow B$ is Borel measurable and $\operatorname{card} f(B)<\infty$. The entropy of $f \in \mathcal{F}$ is defined by

$$
H_{X}(f)=H\left(\mathbb{P}^{f(X)}\right):=-\sum_{a \in f(B)} \mathbb{P}^{X}(f=a) \log \mathbb{P}^{X}(f=a)
$$

Received by the editors December 11, 2003 and, in revised form, June 14, 2004.

2000 Mathematics Subject Classification. Primary 60G15, 94A24; Secondary 60B11, 94A34.

Key words and phrases. Functional quantization, Gaussian process, entropy, distortion rate function. 
(where $0 \log 0:=0$ ). For $H \geq 0$, the $L^{2}$-quantization problem under the entropyconstraint $H$ consists in minimizing

$$
E\|X-f(X)\|^{2}
$$

over all $f \in \mathcal{F}$ with $H_{X}(f) \leq H$. The minimal $H$-quantization error of $X$ is then defined by

$$
e_{H}(X):=\inf \left\{\left(E\|X-f(X)\|^{2}\right)^{1 / 2}: f \in \mathcal{F}, H_{X}(f) \leq H\right\} .
$$

One may check that

$$
\begin{gathered}
e_{H}(X)=\inf \left\{\left(E\|X-f(X)\|^{2}\right)^{1 / 2}: f: B \rightarrow B\right. \text { Borel measurable, } \\
\left.f(B) \text { countable, } H_{X}(f) \leq H\right\} .
\end{gathered}
$$

This notion is obviously related to Shannon's distortion-rate function of $X$ given by

$$
\begin{gathered}
D(H, X):=\inf \left\{\left(\int\|x-y\|^{2} d Q(x, y)\right)^{1 / 2}: Q\right. \text { probability on } \\
B \times B \text { with first marginal } Q_{1}=\mathbb{P}^{X} \text { and } \\
I(Q) \leq H\},
\end{gathered}
$$

where $I(Q)$ denotes the mutual information

$$
I(Q)=\int \log \left(\frac{d Q}{d \mathbb{P}^{X} \otimes Q_{2}}\right) d Q,
$$

the relative entropy of $Q$ relative to the product of the marginals $\mathbb{P}^{X} \otimes Q_{2}$ if $Q$ is absolutely continuous with respect to $\mathbb{P}^{X} \otimes Q_{2}$ and equal to $\infty$ otherwise. Since $I\left(\mathbb{P}^{(X, f(X))}\right)=H_{X}(f)$ for $f \in \mathcal{F}$ (cf. e.g. [5, p. 163]), we have for every $H \geq 0$,

$$
D(H, X) \leq e_{H}(X) .
$$

Throughout all logarithms are natural logarithms.

We address the issue of high-resolution behaviour of $e_{H}(X)$ as $H \rightarrow \infty$. Denote by $K_{X} \subset B$ the reproducing kernel Hilbert space (Cameron-Martin space) associated to $X$. Observe that $\operatorname{supp}\left(\mathbb{P}^{X}\right)$ coincides with the closure of $K_{X}$. Let $\lambda_{1} \geq \lambda_{2} \geq \ldots>0$ be the ordered nonzero eigenvalues of the covariance operator of $X$ (each written as many times as is its multiplicity). The following theorem is devoted to the case $\operatorname{dim} K_{X}=\infty$ and regularly varying eigenvalues. It includes a wide class of Gaussian processes. Recall that a measurable function $\varphi:(s, \infty) \rightarrow(0, \infty)$ $(s \geq 0)$ is said to be regularly varying at infinity with index $b \in \mathbb{R}$ if, for every $t>0$,

$$
\lim _{x \rightarrow \infty} \frac{\varphi(t x)}{\varphi(x)}=t^{b}
$$

Regular variation of $\varphi:(0, s) \rightarrow(0, \infty)(s>0)$ at zero is defined analogously. See [1].

It is convenient to use the symbols $\sim$ and $\lesssim$, where $a_{n} \sim b_{n}$ means $a_{n} / b_{n} \rightarrow 1$ and $a_{n} \lesssim b_{n}$ means $\limsup _{n} a_{n} / b_{n} \leq 1$.

Theorem 1.1. Let $X$ be a centered Gaussian B-valued random vector with dim $K_{X}$ $=\infty$. Assume $\lambda_{j} \sim \varphi(j)$ as $j \rightarrow \infty$, where $\varphi:(s, \infty) \rightarrow(0, \infty)$ is a decreasing, regularly varying function at infinity of index $-b<-1$ for some $s \geq 0$. Then

$$
e_{H}(X) \sim D(H, X) \sim\left(\left(\frac{b}{2}\right)^{b-1} \frac{b}{b-1}\right)^{1 / 2}(H \varphi(H))^{1 / 2} \text { as } H \rightarrow \infty .
$$


Note that the restriction $-b \leq-1$ on the index of $\varphi$ is natural since $\sum_{j=1}^{\infty} \lambda_{j}<$ $\infty$.

Remarks. (a) The most prevalent form for $\varphi$ is

$$
\varphi(x)=c x^{-b}(\log x)^{-a}, b>1, a \in \mathbb{R}, x>\max \left\{1, e^{-a / b}\right\} .
$$

Then we have from the above that

$$
e_{H}(X) \sim\left(c\left(\frac{b}{2}\right)^{b-1} \frac{b}{b-1}\right)^{1 / 2} H^{-(b-1) / 2}(\log H)^{-a / 2} \text { as } H \rightarrow \infty .
$$

(b) The strong equivalence $e_{H}(X) \sim D(H, X)$ is in contrast to the finitedimensional case. If, for instance, $B=\mathbb{R}$ and $\mathbb{P}^{X}=N(0, \lambda), \lambda>0$, then it is known that

$$
D(H, X)=\lambda^{1 / 2} e^{-H}
$$

and

$$
e_{H}(X) \sim\left(\frac{\pi e}{6}\right)^{1 / 2} \lambda^{1 / 2} e^{-H}, H \rightarrow \infty
$$

(cf. [8, Theorem 1.8.7], [16, Theorem 3.1], [17, 7]).

(c) It is enough to consider the centered case since

$$
e_{H}(c X+b)=c e_{H}(X)
$$

whenever $c>0$ and $b \in B$.

Now consider the restriction to Voronoi quantizers defined by

$$
f=\sum_{a \in \alpha} a 1_{C_{a}}
$$

where $\alpha \subset B$, card $\alpha<\infty$ and $\left(C_{a}\right)_{a \in \alpha}$ is a Borel partition of $B$ satisfying

$$
C_{a} \subset\left\{x \in B:\|x-a\|=\min _{b \in \alpha}\|x-b\|\right\}
$$

for every $a \in \alpha$. Set

$e_{H}^{V}(X):=\inf \left\{\left(E\|X-f(X)\|^{2}\right)^{1 / 2}: f \in \mathcal{F}\right.$ Voronoi quantizer, $\left.H_{X}(f) \leq H\right\}$.

Corollary 1.2. Assume the situation of Theorem 1.1. Then

$$
e_{H}(X) \sim e_{H}^{V}(X) \text { as } H \rightarrow \infty .
$$

The precise relationship between the $e_{H}(X)$ problem and the fixed-rate quantization problem can be deduced from the preceding theorem. For $n \in \mathbb{N}$, let

$$
u_{n}(X):=\inf \left\{\left(E\|X-f(X)\|^{2}\right)^{1 / 2}: f \in \mathcal{F}, \operatorname{card} f(B) \leq n\right\} .
$$

Observe that $e_{H}(X) \leq u_{\left[e^{H}\right]}(X)$, where $[x]$ is the integer part of $x \in \mathbb{R}$.

Corollary 1.3. Assume the situation of Theorem 1.1. Then

$$
e_{H}(X) \sim u_{\left[e^{H}\right]}(X) \text { as } H \rightarrow \infty .
$$

The rest of the paper is organized as follows. Section 2 contains some examples. Section 3 is devoted to the proofs. 


\section{EXAMPLES}

We consider centered (componentwise) $L^{2}(\mathbb{P})$-continuous $\mathbb{R}^{k}$-valued Gaussian processes $X=\left(X^{1}, \ldots, X^{k}\right)=\left(X_{t}\right)_{t \in[0,1]^{d}}$. Then $X$ can be seen as centered Gaussian random vector with values in the Hilbert space $B=L_{\mathbb{R}^{k}}^{2}\left([0,1]^{d}, d t\right)$, where $\mathbb{R}^{k}$ is equipped with the Euclidean norm. Its covariance operator is given by

$$
C: B \rightarrow B, C x(t)=\int_{[0,1]^{d}} x(s) \Gamma(s, t) d s,
$$

where $\Gamma(s, t):=\left(E X_{s}^{i} X_{t}^{j}\right)_{i, j \leq k}$ is the covariance function of $X$.

2.1. $k$-dimensional Gaussian diffusions. Let $X=\left(X_{t}\right)_{t \in[0,1]}$ be the unique solution of the equation

$$
d X_{t}^{i}=\sum_{j=1}^{k} A_{i j}(t) X_{t}^{j} d t+d W_{t}^{i}, X_{0}^{i}=0
$$

$i=1, \ldots, k$, where $W$ is a $k$-dimensional standard Brownian motion and $A_{i j} \in$ $L^{2}([0,1], d t)$. Since the eigenvalues of the covariance operator of $W$ satisfy

$$
\lambda_{j}(W) \sim \frac{k^{2}}{\pi^{2}} j^{-2} \text { as } j \rightarrow \infty
$$

and $\mathbb{P}^{X}$ and $\mathbb{P}^{W}$ are equivalent, one gets from the minimax characterization of eigenvalues

Therefore,

$$
\lambda_{j}(X) \sim \lambda_{j}(W) \text { as } j \rightarrow \infty .
$$

$$
e_{H}(X) \sim e_{H}(W) \sim \frac{k \sqrt{2}}{\pi} H^{-1 / 2} \text { as } H \rightarrow \infty .
$$

Note that the dimension $k$ does not effect the rate of convergence to zero of $e_{H}$.

2.2. Fractional Ornstein-Uhlenbeck sheets. The (real) Ornstein-Uhlenbeck sheet on $[0,1]^{d}$ with index $\rho \in(0,2)$ corresponds to

$$
\Gamma(s, t)=\prod_{j=1}^{d} \exp \left(-a_{j}\left|s_{j}-t_{j}\right|^{\rho}\right), a_{j}>0 .
$$

The eigenvalues of its covariance operator satisfy

$$
\lambda_{j} \sim c j^{-(1+\rho)}(\log j)^{(1+\rho)(d-1)} \text { as } j \rightarrow \infty,
$$

where

$$
c=\left(\prod_{j=1}^{d} a_{j}\right)\left(\frac{2 \Gamma(1+\rho) \sin (\pi \rho / 2)}{\pi^{1+\rho}}\right)^{d}((d-1) !)^{-(1+\rho)}
$$

and $\Gamma$ in the above formula denotes the Gamma function (see 11]). Consequently,

$$
e_{H}(\text { FOUS }) \sim\left(c\left(\frac{1+\rho}{2}\right)^{\rho} \frac{1+\rho}{\rho}\right)^{1 / 2} H^{-\rho / 2}(\log H)^{(1+\rho)(d-1) / 2} \text { as } H \rightarrow \infty .
$$

If $\rho=1$, one gets the stationary Ornstein-Uhlenbeck sheet on $[0,1]^{d}$. In this case

$$
e_{H}(\mathrm{OUS}) \sim\left(\prod_{j=1}^{d} a_{j}\right)^{1 / 2} \frac{2^{(d+1) / 2}}{\pi^{d}(d-1) !} H^{-1 / 2}(\log H)^{d-1} \text { as } H \rightarrow \infty .
$$


Remark. The eigenvalue problem has been solved for several other Gaussian processes and other Hilbert spaces including stationary processes with regular highfrequency behaviour of the spectral density, fractional Brownian sheets, Lévy's fractional Brownian motion, integrated Gaussian processes and Gaussian martingales. See [15], [14 p. 79], [1], [2], [9], 3], [12], 13].

\section{Proofs}

A further central object in Shannon's information theory is the rate-distortion function (or $\varepsilon$-entropy) of $X$. It is defined, for $\varepsilon>0$, by

$$
\begin{gathered}
R(\varepsilon, X):=\inf \left\{I(Q): Q \text { probability on } B \times B, Q_{1}=\mathbb{P}^{X},\right. \\
\left.\int\|x-y\|^{2} d Q(x, y) \leq \varepsilon^{2}\right\} .
\end{gathered}
$$

We will rely on the following formula.

Lemma 3.1. Assume the situation of Theorem 1.1 and set $\psi(x):=x \varphi(x), x>s$. Then

$$
R(\varepsilon, X) \sim \frac{b}{2}\left(\frac{b}{b-1}\right)^{1 /(b-1)} \tilde{\psi}\left(1 / \varepsilon^{2}\right) \text { as } \varepsilon \rightarrow 0,
$$

where $\tilde{\psi}$ is the (up to strong equivalence $\sim$ ) uniquely determined function which is regularly varying at infinity with index $1 /(b-1)$ such that

$$
\tilde{\psi} \circ \frac{1}{\psi}(x) \sim \frac{1}{\psi} \circ \tilde{\psi}(x) \sim x \text { as } x \rightarrow \infty .
$$

In particular, $R(\cdot, X)$ is regularly varying at zero with index $-2 /(b-1)$.

Proof. This is an easy consequence of the "flooding" formula for $R(\varepsilon, X)$, due to Kolmogorov and Pinsker, and regular variation of the eigenvalues. See the proof of Corollary 2.4 in [1].

Proof of Theorem 1.1. Set $D(H)=D(H, X), e_{H}=e_{H}(X)$ and $R(\varepsilon)=R(\varepsilon, X)$. Observe first that $\lim _{H \rightarrow \infty} e_{H}=0$. Furthermore, $D(0)=\left(E\|X\|^{2}\right)^{1 / 2}, D(H)>0$ and

$$
R(D(H))=H
$$

for every $H \in \mathbb{R}_{+}$(cf. [8, Theorem 1.7.2]). For $\varepsilon>0$, let

$$
\begin{array}{ccc}
N(\varepsilon)=N(\varepsilon, X):= & \min \left\{n \in \mathbb{N}: u_{n}(X) \leq \varepsilon\right\} \\
= & \min \{n \in \mathbb{N}: \exists f \in \mathcal{F},|f(B)| \leq n \text { such } \\
& \text { that } \left.E\|X-f(X)\|^{2} \leq \varepsilon^{2}\right\} .
\end{array}
$$

Then by Corollary 2.4 in Luschgy and Pagès [11,

$$
R(\varepsilon) \sim \log N(\varepsilon) \text { as } \varepsilon \rightarrow 0 .
$$

Moreover, we have for every $H \in \mathbb{R}_{+}, c \in(0,1)$,

$$
H \leq \log N\left(c e_{H}\right) \text {. }
$$

In fact, if $f \in \mathcal{F}$, card $f(B) \leq n$ such that $\left(E\|X-f(X)\|^{2}\right)^{1 / 2} \leq c e_{H}$, then $\log n \geq H_{X}(f)>H$. This gives (3.2). Using (1.4) one thus obtains

$$
R\left(e_{H}\right) \leq R(D(H))=H \leq \log N\left(c e_{H}\right)
$$

and hence

$$
R\left(e_{H}\right) \leq R(D(H)) \lesssim R\left(c e_{H}\right) \sim c^{-2 /(b-1)} R\left(e_{H}\right), H \rightarrow \infty .
$$


The latter strong equivalence follows from regular variation of $R$ at zero with index $-2 /(b-1)$ (see Lemma 3.1). Letting $c \rightarrow 1$ yields

$$
R\left(e_{H}\right) \sim R(D(H)) \text { as } H \rightarrow \infty .
$$

Finally, by Lemma 3.1,

$$
\frac{1}{\psi}(R(\varepsilon)) \sim\left(\frac{b}{2}\right)^{b-1} \frac{b}{b-1} \varepsilon^{-2}
$$

and therefore

$$
\varepsilon \sim\left(\left(\frac{b}{2}\right)^{b-1} \frac{b}{b-1}\right)^{1 / 2}(\psi(R(\varepsilon)))^{1 / 2} \text { as } \varepsilon \rightarrow 0 .
$$

Using (3.3), this implies

$$
e_{H} \sim D(H) \text { as } H \rightarrow \infty
$$

and

$$
D(H) \sim\left(\left(\frac{b}{2}\right)^{b-2} \frac{b}{b-1}\right)^{1 / 2} \psi(H)^{1 / 2} \text { as } H \rightarrow \infty .
$$

Proof of Corollaries 1.2 and 1.3. One derives

$$
e_{H}(X) \sim u_{\left[e^{H}\right]}(X) \text { as } H \rightarrow \infty
$$

from Theorem 1.1 and [11, Theorem 2.2]. Let $u_{n}^{V}(X)$ be defined analogously to (1.5). Then $u_{n}(X)=u_{n}^{V}(X)$ and hence, for every $H \geq 0$

$$
e_{H}(X) \leq e_{H}^{V}(X) \leq u_{\left[e^{H}\right]}(X) .
$$

This yields the assertions.

\section{REFERENCES}

[1] Bingham, N.H., Goldie, C.M. and Teugels, J.L. (1987). Regular Variation. Cambridge University Press. MR 0898871|(88i:26004)

[2] Bronski, J.C. (2003). Small ball constants and tight eigenvalue asmptotics for fractional Brownian motions. J. Theoretical Probab. 16, 87-100. MR1956822 (2004b:60105)

[3] Gao, F., Hanning, J. and Torcaso, F. (2003). Integrated Brownian motions and exact $L^{2}$-small balls. Ann. Probab. 31, 1320-1337. MR1989435 (2004k:60104)

[4] Gersho, A. and Gray, R.M. (1992). Vector Quantization and Signal Compression. Kluwer, Boston.

[5] Graf, S. and Luschgy, H. (2000). Foundations of Quantization for Probability Distributions. Lecture Notes in Math. 1730. Springer, Berlin. MR1764176 (2001m:60043)

[6] Gray, R.M. and Neuhoff, D.L. (1998). Quantization. IEEE Trans. Inform. Theory 44, 2325 2383. MR.1658787 (99i:94029)

[7] Gray, R.M., Linder, T. and Li, J. (2002). A Lagrangian formulation of Zador's entropyconstrained quantization theorem. IEEE Trans. Inform. Theory 48, 695-707. MR1889976 (2003f:94036)

[8] Ihara, S. (1993). Information Theory for Continuous Systems. World Scientific, Singapore. MR.1249933 (94m:94008)

[9] Karol', A.I., Nazarov, A.I. and Nikitin, Ya. Yu. (2003). Tensor products of compact operators and logarithmic $L^{2}$-small ball asymptotics for Gaussian random fields. Preprint.

[10] Luschgy, H. and Pagès, G. (2002). Functional quantization of Gaussian processes. J. Funct. Anal. 196, 486-531. MR.1943099 (2003i:60006)

[11] Luschgy, H. and Pagès, G. (2004). Sharp asymptotics of the functional quantization problem for Gaussian processes. Ann. Probab. 32, 1574-1599. MR 2060310

[12] Nazarov, A.I. and Nikitin, Ya. Yu. (2004). Exact $L^{2}$-small ball behaviour of integrated Gaussian processes and spectral asymptotics of boundary value problems. Probab. Theory Related Fields 129, 469-494. MR2078979 
[13] Nazarov, A.I. and Nikitin, Ya.Yu. (2003). Logarithmic $L^{2}$-small ball asymptotics for some fractional Gaussian processes. Preprint.

[14] Ritter, K. (2000). Average-Case Analysis of Numerical Problems. Lecture Notes in Math. 1733. Springer, Berlin. MR 1763973 (2001i:65001)

[15] Rosenblatt, M. (1963). Some results on the asymptotic behaviour of eigenvalues for a class integral equations with translation kernel. J. Math. and Mechanics 12, 619-628. MR0150551 $(27: 547)$

[16] Zador, P.L. (1963). Development and evaluation of procedures for quantizing multivariate distributions. Ph.D. dissertation, Stanford Univ.

[17] Zador, P.L. (1966): Topics in the asymptotic quantization of continuous random variables. Bell Laboratories Technical Report.

Fakultät für Mathematik und Informatik, Universität Passau, D-94030 Passau, GerMANY

E-mail address: graf@fmi.uni-passau.de

FB IV-Mathematik, Universität Trier, D-54286 Trier, Germany

E-mail address: luschgy@uni-trier.de 